\title{
Segmentação das áreas pulmonares em radiografias torácicas digitais
}

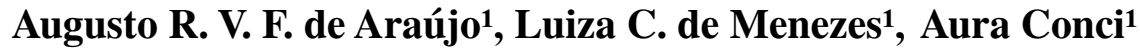 \\ ${ }^{1}$ Instituto de Computação - Universidade Federal Fluminense (UFF) \\ 24.210-346 - Niterói - RJ - Brasil \\ augustorighetti@id.uff.br, lumenezesdid.uff.br, aconci@id.uff.br
}

\begin{abstract}
Radiography has become an indispensable tool to aid medical diagnosis. Although other techniques are available, radiographs are accessible, fastly acquired and used on a large scale. This work presents an automatic method for lung regions segmentation in chest $X$-ray and utilizes simple image processing techniques. The developed ground truths are publicly available; results compared to previous reports, and usable in computer-aided diagnosis systems.
\end{abstract}

Resumo. Radiografias são ferramentas indispensáveis para auxílio ao diagnóstico médico. Ainda que outras técnicas estejam disponíveis, a radiografia é um exame acessível, de rápida aquisição e utilizado em larga escala. Este trabalho apresenta um método de segmentação automatizada das áreas pulmonares em radiografias torácicas e para tal, faz uso de técnicas de processamento de imagens simples. Disponibilizamos publicamente as segmentações desenvolvidas; resultados são comparados aos de outras técnicas disponíveis na literatura e podem ser utilizados como entrada em sistemas de auxílio ao diagnóstico.

\section{Introdução}

Nos últimos anos, a análise de imagens radiográficas digitais tem sido utilizada como uma ferramenta indispensável à investigação clínica. Mesmo a radiografia convencional tem papel importante por ser um exame relativamente acessível, rápido e viável para uso em larga escala.

Para realizar a identificação de manifestações patológicas, ou mesmo sequelas de patologias pregressas, o médico interpreta a imagem radiográfica gerada identificando as estruturas anatômicas 3D projetadas em um plano bidimensional, portanto, sobrepostas. Esta sobreposição se deve ao processo de aquisição, onde as estruturas que estão paralelas à direção da incidência dos raios-X são projetadas em um plano bidimensional e representadas em escala de cinza de acordo com a densidade de cada tecido que os raios-X penetram. A quantidade de raio- $X$ que consegue atravessar as estruturas anatômicas pode ser variável e depende da escolha do tempo de exposição, kilovoltagem e miliamperes (kV e mA) utilizados (Krupinski et al., 2007; Soares, 2008).

A análise radiográfica feita pelo médico radiologista normalmente é realizada em etapas, onde a imagem é intuitivamente segmentada, de modo que cada um dos componentes da imagem radiográfica é analisado separadamente, e por fim em conjunto, para a elaboração da impressão diagnóstica. A segmentação das áreas pulmonares pode ser então, considerada fundamental no processo de diagnóstico radiológico, tendo em vista que facilita análises relacionadas a: (1) insuflação pulmonar (normoinsuflação, hiperinsuflação e hipoinsuflação); (2) identificação de bordas (a 
serem usadas no cálculo do índice cardiotorácico, por exemplo); (3) identificação de achados radiográficos periféricos (placas pleurais calcificadas, pneumotórax, etc.); e outras (Goodman e Felson, 2014; Corne e Kumaran, 2016). Em termos de análises computacionais, a segmentação pulmonar pode ser considerada uma etapa de pré-processamento determinante para o desempenho dos algoritmos que avaliam doenças que afetam essa região, como doenças do interstício e do parênquima pulmonar.

\subsection{Objetivos}

Este estudo considera a segmentação das áreas pulmonares em imagens radiográficas, tendo em vista que esta tarefa pode ser utilizada como etapa de pré-processamento para definição da região de interesse a ser utilizada em análises que auxiliem o diagnóstico de doenças pulmonares, como por exemplo: bronquite, enfisema, câncer de pulmão, tuberculose e todos os tipos de pneumonias (Lacerda et al, 2021).

Nesse sentido, este trabalho tem a proposta de, dada uma imagem de radiografia torácica adquirida em posição ortostática (i.e. em que o paciente está em posição vertical), com incidência posteroanterior (PA) (quando os raios- $\mathrm{X}$ incidem nas costas do paciente e a película de captação está situada à frente deste), gerar uma imagem binária que represente a área pulmonar correlata por meio de técnicas de computação visual. A proposta é que trabalhos futuros possam utilizar este resultado como máscara ou região de interesse, conhecida em inglês como Region of Interest (ROI). Cabe ressaltar que essa combinação de posição e incidência é a mais comumente utilizada, enquanto radiografias com incidência anteroposterior ou em posição dorsal são reservadas para casos particulares, como pacientes acamados ou investigação de alterações específicas.

\subsection{Estrutura do artigo}

As seções a seguir estão organizadas em (2) Trabalhos Relacionados, (3) Metodologia, (4) Resultados e (5) Considerações Finais. A seção Trabalhos Relacionados se destina à apresentação de técnicas encontradas na literatura com finalidade similar ao objetivo em questão. Na seção Metodologia é apresentado o método desenvolvido. A seção Resultados aborda os resultados obtidos e alguns aspectos comparativos com resultados obtidos por trabalhos anteriores. Finalmente, a seção Considerações Finais indica caminhos de prosseguimento deste trabalho.

\section{Trabalhos Relacionados}

Recentemente Rahman et al. (2020) realizaram três experimentos relacionados à detecção automatizada de tuberculose pulmonar em radiografias torácicas. O primeiro dos experimentos apresentados é o de segmentação das áreas pulmonares utilizando redes neurais convolucionais do tipo U-net. Neste experimento foram comparados os resultados de duas redes U-net e a rede com melhor desempenho foi utilizada para realizar a segmentação das imagens dos experimentos subsequentes realizados pela equipe.

Outro experimento importante realizado no trabalho de Rahman et al. (2020) foi a comparação do desempenho de diversas redes neurais quando se utilizava como 
entrada as imagens originais e imagens contendo apenas as áreas pulmonares segmentadas. Invariavelmente à escolha da rede neural, quando a entrada continha apenas as áreas pulmonares segmentadas, o desempenho era superior, demonstrando a importância da segmentação das áreas pulmonares como passo de pré-processamento neste caso.

Por outro lado, Saad et al. (2014) buscaram solucionar o problema da segmentação pulmonar por meio de técnicas de processamento de imagens. Os autores sinalizaram que o uso de técnicas de detecção de bordas em imagens de radiografia de tórax é parte essencial do processo de segmentação pulmonar porque as bordas são as estruturas responsáveis por representar as características de contorno da imagem. Nesse sentido, os autores propuseram uma solução que envolve a utilização de um filtro Canny para a binarização da imagem seguido das operações morfológicas de dilatação e erosão. Para desenvolvimento do algoritmo, os autores utilizaram o software Matlab 2012a e um total de 10 imagens do conjunto de dados da Japan Society of Radiological Technology, que é público e contém 247 imagens, além de informações adicionais em formato de texto sobre o caso retratado pela radiografia, como idade e gênero do paciente. Caso a imagem contenha nódulo, são também informados o diagnóstico patológico (benigno/maligno) e a respectiva posição na imagem (Saad et al., 2014).

Para fins de avaliação do método proposto por Saad et al., os autores criaram manualmente imagens binárias contendo a segmentação das áreas pulmonares (esquerda e direita) correspondentes. Tais imagens, chamadas Ground Truths (GTs), foram utilizadas como referência para cálculo do índice de similaridade das saídas do modelo. O índice de similaridade escolhido pelos autores foi o índice de Jaccard devido ao fato de que o valor deste coeficiente pode ser interpretado com uma explicação simples em que quanto mais alto mais próxima a máscara de saída é comparável à imagem GT. Por outro lado, um valor mais baixo significa que a semelhança da saída ao GT é inferior. O coeficiente de Jaccard médio obtido para as 10 imagens (considerando ambas as áreas pulmonares) foi de 0,809 . Os autores afirmam, no entanto, que outros estudos, utilizando a mesma base de dados, obtiveram resultados médios ainda mais altos como 0,954 (Candemir et al, 2013) e 0,950 (Van Ginneken et al, 2006).

Em Candemir et al. (2013), os autores criaram um processo de correspondência de imagens. Isto é, ao receber a imagem de um novo paciente, o algoritmo procura uma imagem similar na base de entrada e segue o cálculo com a referência do modelo pulmonar identificado, aplicando o mapeamento de transformação às máscaras de treinamento. Finalmente, a imagem binária segmentada é obtida por meio da soma média das máscaras transformadas.

Em relação ao estudo de Van Ginneken et al. (2006), foram comparados três métodos de segmentação supervisionados: (1) modelos de forma ativa; (2) modelos de aparência ativa; e (3) classificação de pixels. O método que apresentou melhor resultado foi o de classificação de pixels, tendo sido quantitativamente superior ao de um observador humano. Este método considera a computação de uma série de características da amostra, como níveis de cinza e filtros, para utilização em uma rede do tipo $k$-Nearest Neighbors $(\mathrm{kNN})$, que classifica a imagem conforme os "k" vizinhos mais próximos. 
Verifica-se, portanto, que os estudos prévios apresentados fazem uso de técnicas complexas, alimentadas por um número elevado de imagens e dependentes de especialistas para a marcação prévia das áreas de interesse. Adicionalmente, observa-se que fabricantes como a Philips (2021) e a GE Healthcare (2020) têm atuado no desenvolvimento de produtos com processo automatizado de escolha de parâmetros com o intuito de melhorar a definição e contraste das imagens radiográficas que, consequentemente, facilitam a segmentação das regiões pulmonares em imagens de radiografias torácicas. No entanto, tais iniciativas têm um custo associado e se tornam inviáveis para a realidade de muitas clínicas e hospitais. Nesse sentido, o trabalho aqui proposto visa a utilização de processamentos menos custosos sem a necessidade de bases de dados prévias para obtenção de resultados similares.

\section{Metodologia}

O método proposto foi implementado em Python v3.7 e fez uso das bibliotecas Scikit Image (van der Walt et al., 2014) e OpenCV. Para comparação com trabalhos anteriores, a base de imagens escolhida foi a fornecida pela Japan Society of Radiological Technology (JSRT). Esta base é pública e gratuita, com resolução de 2048 x 2048 pixels, em formato (loseless) IMG (.img) de 12 bit (escala de cinza/4096). Os autores da base citam que as imagens foram utilizadas em contexto real de avaliação dos pacientes em questão. É importante mencionar que os Ground Truths (GTs) de Saad et al. (2014) não puderam ser localizados, tendo sido elaborada uma nova base de GT pelo médico especialista da equipe, que está disponível para download em https://github.com/ augustorighetti/JSRT-DB_GroundTruth.

O fluxograma do método proposto por este artigo é observado na Figura 1.

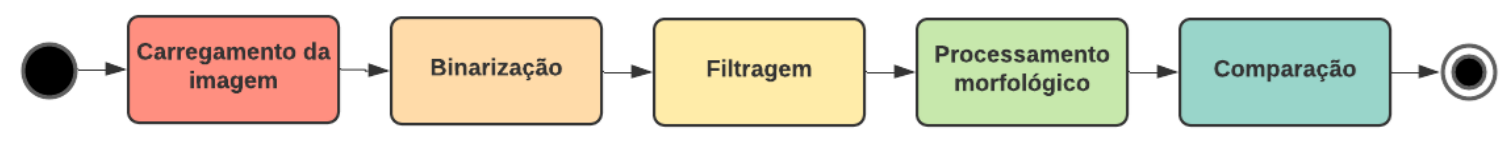

Figura 1. Fluxograma do método proposto
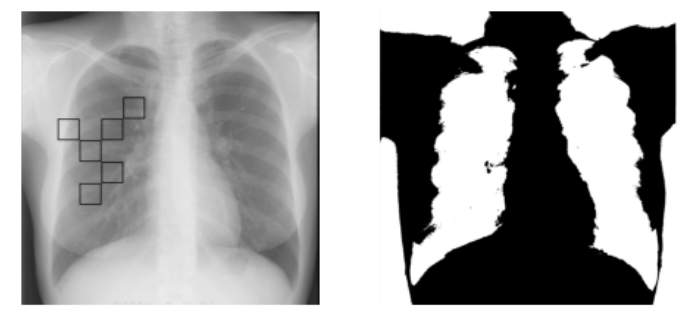

Figura 2. À esquerda, JPCNN001 em 256 tons com marcações representando as regiões a serem usadas para identificação do limiar de binarização. À direita, a imagem após a binarização proposta.

Após carregar a imagem, um processo de binarização foi desenvolvido. Este processo considera seis (6) áreas quadradas, ilustradas na Figura 2 à esquerda, que 
individualmente correspondem a cerca de $0,5 \%$ da área da imagem, para definição do limiar de binarização.

Observou-se a necessidade do desenvolvimento de um método de binarização, pois, os histogramas das imagens de radiografias torácicas utilizadas neste trabalho não apresentaram uma distribuição onde houvesse um tom de cinza adequado entre as modas a ser definido como um limiar na binarização (isto é, um tom que seja associável a uma possível separação das áreas a serem incluídas nos conjuntos de cores preto e branco) por todos os métodos já desenvolvidos na literatura (Sonka et al, 2007). Isto acontece porque a região de interesse contém pontos com intensidades de cinza muito similares ao fundo da imagem como pode ser verificado no histograma representado na Figura 3, em que para o caso exemplificado o valor adequado para a limiarização seria entre 0,2 e 0,3 .

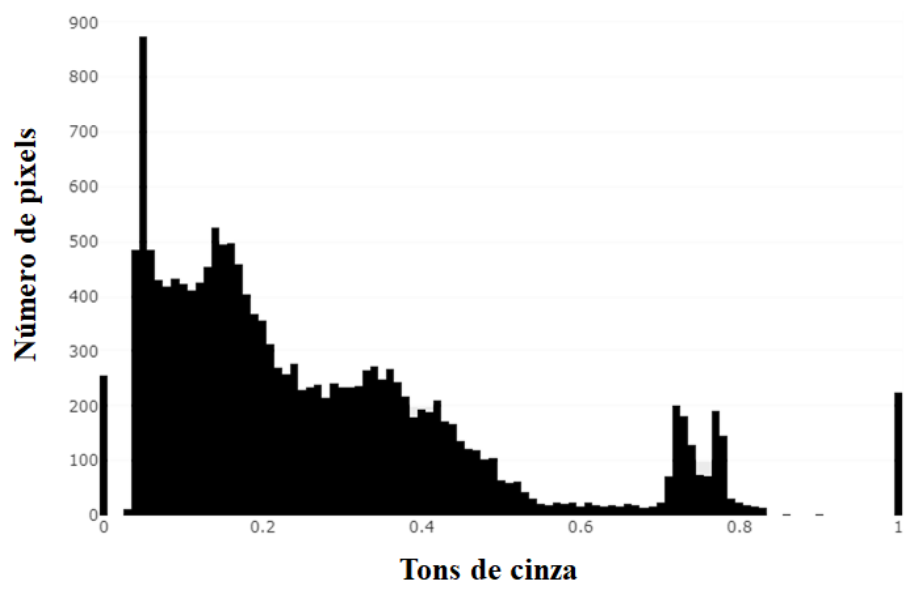

Figura 3. Histograma de JPCLN006

A escolha da posição e do número das regiões quadradas mostradas na Figura 2, bem como suas dimensões, foi feita baseada em experimentações utilizando radiografias com áreas pulmonares de dimensões variadas e com pacientes de diferentes idades e gêneros. Nesse sentido, procurou-se generalizar o método por meio da escolha de seis (6) regiões com tamanho significativo o suficiente para conter a informação necessária à binarização e ao mesmo tempo se manterem internas à região de interesse. Para isso, as áreas das marcações mencionadas foram distribuídas em relação ao centro da imagem em uma região que corresponde ao hemitórax direito. A escolha da localização dessas áreas foi baseada em duas premissas: a primeira é que na maior parte da população a área cardíaca estará predominantemente projetada no hemitórax esquerdo; a segunda é de que radiografias deste tipo precisam que o paciente esteja posicionado corretamente, isto é, de forma aproximadamente centralizada à imagem. Desse modo, a maioria das áreas delimitadas invariavelmente estarão localizadas em região pulmonar (região de interesse) e não na região cardíaca ou da coluna vertebral.

Após diversos experimentos chegou-se, portanto, a um processo simples e eficiente. O processo utilizado consiste em: (1) obter o tom de cinza máximo dos pixels de cada uma destas seis áreas/marcações; (2) descartar os valores de máximo e o 
mínimo dentre os 6 valores; e, por fim, (3) calcular a média dos quatro valores restantes, que será utilizada como limiar $m$ da binarização. O descarte dos valores extremos (máximo e mínimo) da lista de máximos encontrados é uma forma de tentar evitar valores fora do esperado (outliers), que podem ocorrer, principalmente, quando há um deslocamento do paciente em relação ao centro da imagem. Nesse sentido, o descarte foi utilizado para garantir maior generalização do processo de binarização. Finaliza-se o processo de binarização considerando a média $(m)$ de modo que:

$$
\text { pixel_binarizado }_{i, j}=\left\{\begin{array}{l}
1, \text { caso pixel_original }_{i, j} \geq m \\
0, \text { caso pixel_original }_{i, j}<m
\end{array}\right.
$$

em que pixel_binarizado ${ }_{i, j}$ representa o novo valor do pixel nas coordenadas $i, j$ da imagem binarizada e pixel_original ${ }_{i, j}$ representa o tom do pixel nas mesmas coordenadas $i, j$, porém da imagem original. A Figura 2 à direita mostra como ficou a imagem inicial (à esquerda) após a binarização proposta.

Por se tratar de um banco de imagens radiográficas digitalizadas, algumas radiografias apresentam ruídos inerentes ao processo de digitalização, principalmente nas regiões periféricas da borda. Para minimizar os efeitos deletérios desses artefatos, todos os pixels que distam 50 pixels dos limites da imagem são substituídos por valor 0 antes de prosseguir.

Uma vez que o objetivo final é segmentar apenas as áreas pulmonares, qualquer tom não zero fora da região pulmonar ao final do processamento seria considerado ruído pela abordagem avaliativa utilizada. Para remover as áreas brancas que não são de interesse, i.e. toda região externa à imagem do corpo, foi realizada uma varredura vertical a partir do pixel no canto superior esquerdo da imagem (que normalmente é um pixel branco) até encontrar um pixel que representasse o corpo do paciente (pixel preto). À medida em que essa varredura é realizada, os pixels brancos são substituídos por pretos. Abordagem semelhante foi utilizada para remoção do plano de fundo nas porções inferolaterais. A Figura 4 ilustra essa varredura e o preenchimento do fundo (das porções superiores).

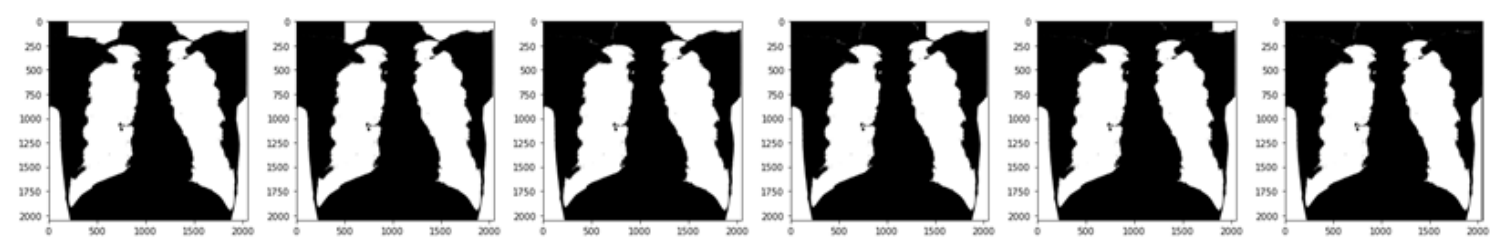

Figura 4. Sequência representativa de parte do processo de preenchimento

Após a abordagem de preenchimento proposta, a imagem obtida ainda contém resquícios fora da área pulmonar, como ilustrado na Figura 5, cujos detalhes estão mostrados invertidos em relação ao tom de cinza para melhor visualização. 


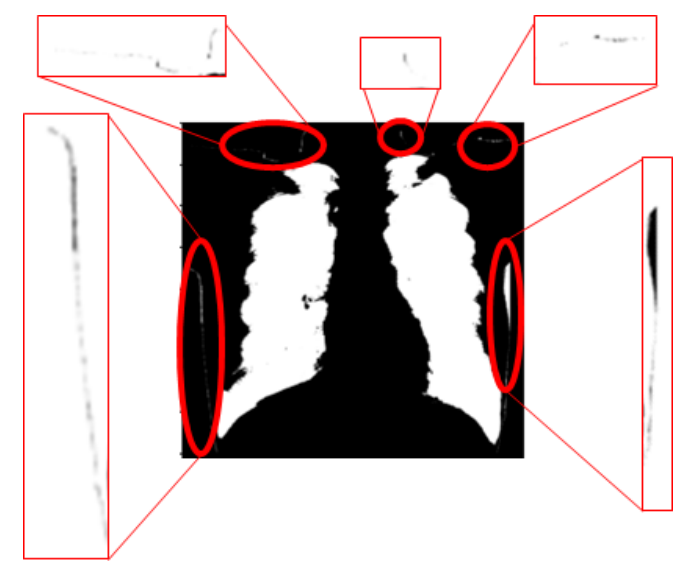

Figura 5. Resultado do preenchimento utilizando varredura vertical

Para minimizar estes ruídos, a imagem binarizada inicial é subtraída pela imagem preenchida, obtendo assim uma máscara, onde está apenas o ruído identificado na imagem binarizada - representado na Figura 6 (c). Sobre esta máscara é aplicada a operação morfológica de dilatação utilizando três elementos estruturantes ${ }^{1}$ : disco com raio 60 e retângulos com dimensões 30 x 10 e 10 x 30. Por fim, este resultado é subtraído da imagem binarizada inicial. Este processo é ilustrado na Figura 6.

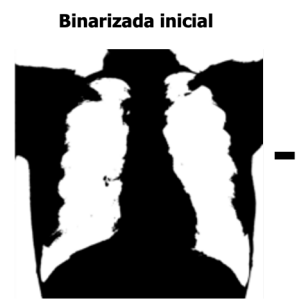

(a)

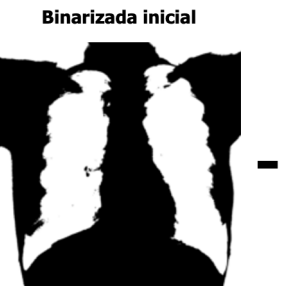

(d)
Preenchida

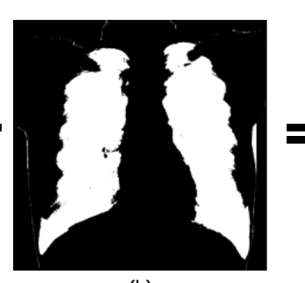

(b)

Ruído dilatado

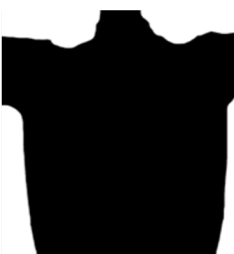

(e)

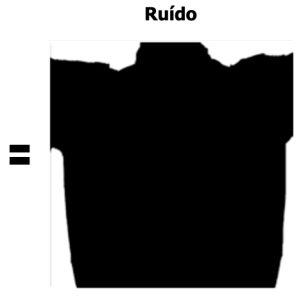

(c)

Subtraída

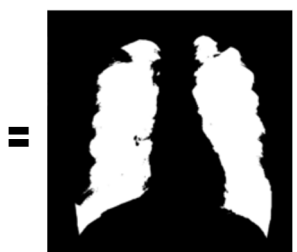

(f)

Figura 6. Etapas de definição da ROI

$\mathrm{Na}$ sequência, para garantir que o contorno externo da região pulmonar na imagem subtraída seja mais próximo do contorno esperado, além de garantir que não haja "buracos", i.e. falhas de conexão entre os pixels da ROI, são aplicadas operações morfológicas à imagem subtraída. Foram utilizadas operações de fechamento com um quadrado (lado de 35) seguida de um disco (raio de 35) como elementos estruturantes. O objetivo principal destas operações é reduzir a quantidade de pequenos ruídos

\footnotetext{
${ }^{1} \mathrm{O}$ algoritmo desenvolvido recalcula para qualquer resolução de entrada o tamanho destes elementos proporcionalmente às dimensões da imagem de entrada. Para fins de simplificação, as dimensões dos elementos estruturantes são apresentadas ao longo do texto em número de pixels proporcionais às dimensões do banco de dados utilizado, i.e. 2048x2048.
} 
concentrados principalmente ao redor da silhueta cardíaca e ao longo das áreas laterais dos pulmões. A Figura 7 (centro) mostra o resultado em uma das imagens utilizadas.
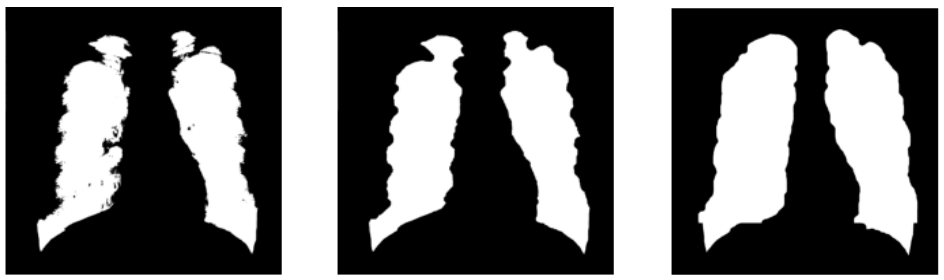

Figura 7. Sequência de resultados das operações morfológicas

Observa-se que as porções inferiores da ROI, correspondentes à cúpula diafragmática e bases pulmonares, apresentam visualmente aspecto satisfatório. Por outro lado, a definição dos contornos laterais fica comprometida por apresentar alta sensibilidade ao processo de binarização. Nesse sentido, são empregados operadores de dilatação e fechamento em $80 \%$ da altura da imagem, i.e. acima da região correspondente às bases pulmonares (região próxima às cúpulas diafragmáticas). Foram realizadas duas dilatações com os seguintes elementos estruturantes: retângulo (altura de 40 e largura de 2) e octógono regular (com lados de 17). Por fim, uma operação de fechamento utilizando o elemento estruturante disco (com raio de 25) para suavizar ângulos presentes nas bordas. O resultado dessas dilatações está representado na Figura 7 à direita.

O resultado do método proposto é uma máscara binária em que os pixels com valor um (1) representam as áreas de interesse.

\section{Resultados}

As imagens resultantes do processamento foram comparadas às segmentações manuais (imagens GT) pixel a pixel para quantificar a acurácia do método proposto. A análise comparativa foi feita em cada uma das dez (10) imagens. Por fim, foi realizada uma média dos valores das comparações para obtenção da matriz confusão, indicada na Tabela 1.

Tabela 1. Matriz de confusão das médias das dez comparações realizadas

\begin{tabular}{|c|c|c|c|}
\cline { 3 - 4 } \multicolumn{2}{c|}{} & \multicolumn{2}{c|}{ Valor encontrado (ROI resultante) } \\
\cline { 3 - 4 } \multicolumn{2}{c|}{} & Pixel branco (1) & Pixel preto (0) \\
\hline \multirow{2}{*}{$\begin{array}{l}\text { Valor esperado } \\
\text { (ground truth) }\end{array}$} & Pixel branco (1) & 0,287 & 0,038 \\
\cline { 2 - 4 } & Pixel preto (0) & 0,045 & 0,630 \\
\hline
\end{tabular}

A partir desses valores, calcularam-se as medidas de desempenho mostradas na Tabela 2, que auxiliam na avaliação quantitativa do resultado obtido. 
Tabela 2. Medidas de desempenho médias das dez comparações realizadas

\begin{tabular}{|l|l|c|}
\hline Avaliador & Definição & Valor obtido \\
\hline Acurácia & $(\mathrm{TP}+\mathrm{TN}) /(\mathrm{TP}+\mathrm{TN}+\mathrm{FP}+\mathrm{FN})$ & 0,917 \\
\hline Sensibilidade & $\mathrm{TP} /(\mathrm{TP}+\mathrm{FN})$ & 0,883 \\
\hline Especificidade & $\mathrm{TN} /(\mathrm{TN}+\mathrm{FP})$ & 0,933 \\
\hline F1 Score & 2TP/(2TP + FP + FN) & 0,873 \\
\hline Índice de Jaccard & $\mathrm{TP} /(\mathrm{TP}+\mathrm{FP}+\mathrm{FN})$ & 0,777 \\
\hline
\end{tabular}

A média do índice de Jaccard obtida por Saad et al (2014) foi de 0,809, já a média obtida por Rahman et al (2020) foi de 0,924. A abordagem apresentada neste artigo obteve média de 0,777 , entretanto utilizando recursos computacionalmente menos onerosos. A Figura 8 apresenta as imagens originais, os GTs e os resultados das imagens após a abordagem proposta.
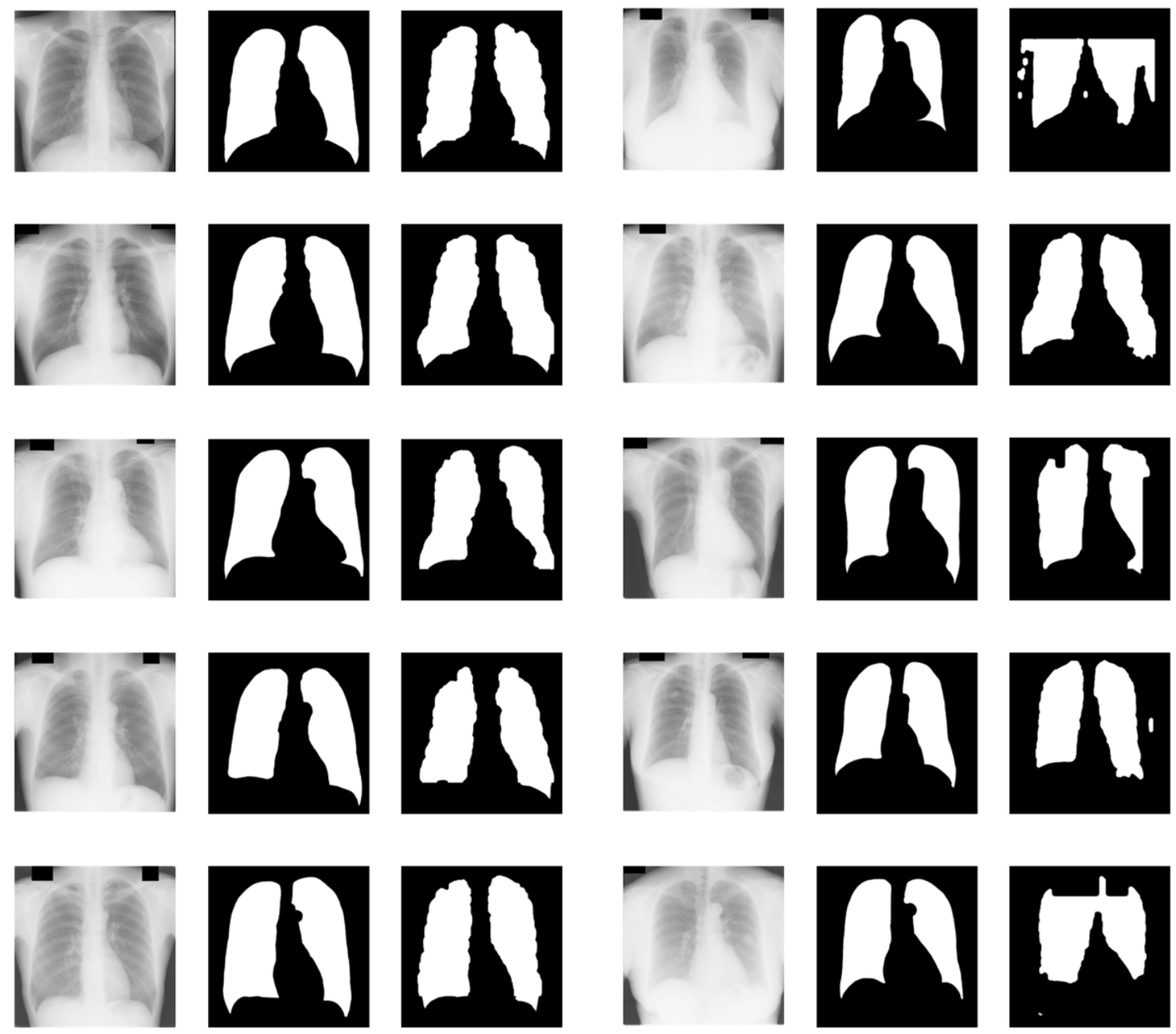

Figura 8. Imagens originais, GTs e resultados finais, respectivamente 


\section{Considerações Finais}

Este trabalho apresenta um método simples e eficiente de segmentação pulmonar em radiografias torácicas em PA. A utilização do método ao invés da segmentação manual das áreas pulmonares torna o processamento mais rápido, reprodutível e não suscetível à subjetividade do operador. Nesse sentido, observa-se que as laterais das áreas pulmonares são mais difíceis de serem delimitadas, uma vez que há maior sobreposição de tecido adjacente nessas regiões, como as curvas das costelas. Adicionalmente, destaca-se que patologias no pulmão esquerdo poderiam comprometer a identificação dos contornos da silhueta cardíaca, por exemplo.

O método proposto por este trabalho utiliza métodos simples, de baixo custo computacional, de fácil replicação e sem a necessidade de um banco de imagens previamente segmentadas para ser implementado. Entretanto, entende-se que melhorias, principalmente no que diz respeito a uma maior generalização dos tipos de imagens de entrada, como em casos de imagens com baixo grau de contraste, podem ser desenvolvidas.

Próximos passos neste sentido envolvem: uma abordagem sem utilizar a limiarização, isto é, com uso das imagens nos níveis de cinza originais da base de imagens; aumento do número de GTs; e comparações com trabalhos que tenham outros índices de avaliação além do de Jaccard (apresentado por Saad et al., 2014) para avaliações mais abrangentes.

O código-fonte e GTs apresentados neste artigo estão disponíveis em https://github.com/augustorighetti/JSRT-DB_GroundTruth.

\section{Agradecimentos}

A.R. e L.M. agradecem à CAPES pelo apoio em seu mestrado no IC/UFF. A.C. é apoiada parcialmente pelo projeto MACC-INCT, pelo CNPq (projetos 402988 / 2016-7 e 305416 / 2018-9) e pela FAPERJ (projetos SIADE-2, e-Health Rio e Digit3D).

\section{Referências}

Candemir, S., Jaeger, S., Palaniappan, K., Musco, J. P., Singh, R. K., Xue, Z. \& McDonald, C. J. (2013), Lung segmentation in chest radiographs using anatomical atlases with nonrigid registration. IEEE Transactions on Medical Imaging, 33(2), 577-590.

Corne, J. \& Kumaran, M. (2016), Chest x-ray made easy. 4th ed. Edinburgh, UK: Elsevier.

General Electric (2020). A new frontier in digital X-ray image quality. G Healthcare Products. Disponível em: <https://www.gehealthcare.com.br/en-CA/products/ radiography/fixed-rad-systems/discovery-xr656-hd-x-ray-system-powered-by-helix>. Acesso em 28/03/2021.

Goodman, L. \& Felson, B. (2014), Felson's Principles of Chest Roentgenology, A Programmed Text. 4th ed. Philadelphia, PA: Elsevier, Saunders. 
Krupinski, E. A., Williams, M. B., Andriole, K., Strauss, K. J., Applegate, K., Wyatt, M., Bjork, S. \& Seibert, J. A. (2007). Digital radiography image quality: image processing and display. Journal of the American College of Radiology, 4(6), 389-400.

Lacerda P, Barros B, Albuquerque C \& Conci A. (2021), Hyperparameter Optimization for COVID-19 Pneumonia Diagnosis Based on Chest CT. Sensors; 21(6), 2174. https://doi.org/10.3390/s21062174.

Philips (2021). Next generation image processing. USA Philips Healthcare Resources. Disponível em: <https://www.usa.philips.com/healthcare/resources/landing/unique-X -ray>. Acesso em 28/03/2021.

Saad, M.N., Muda, Z., Ashaari, N. S., \& Hamid, H. A. (2014), Image segmentation for lung region in chest X-ray images using edge detection and morphology. In 2014 IEEE International Conference on Control System, Computing and Engineering (ICCSCE 2014), 46-51.

Shiraishi J, Katsuragawa S, Ikezoe J, Matsumoto T, Kobayashi T, Komatsu K, Matsui M, Fujita H, Kodera Y, \& Doi K. (2000), Development of a digital image database for chest radiographs with and without a lung nodule: Receiver operating characteristic analysis of radiologists' detection of pulmonary nodules. American Journal of Radiology 174, 71-74.

Soares, J.A.C.R. (2008), Princípios básicos de física em radiodiagnóstico. - 2. ed. rev. São Paulo: Colégio Brasileiro de Radiologia. disponível em: https://cbr.org.br/ wp-content/uploads/2019/06/Apostila-de-Fisica_2008.pdf.

Sonka, M., Hlavac, V., and Boyle, R. (2007). Image Processing, Analysis, and Machine Vision. ThomsonEngineering.

Van der Walt, S., Schönberger, J.L., Nunez-Iglesias, J., Boulogne, F., Warner, J.D., Yager, N., Gouillart, E. \& Yu, T. (2014), Scikit-image: Image processing in Python. PeerJ 2:e453 https://doi.org/10.7717/peerj.453.

Van Ginneken, B., Stegmann, M. B., \& Loog, M. (2006), Segmentation of anatomical structures in chest radiographs using supervised methods: a comparative study on a public database. Medical Image Analysis, 10(1), 19-40.

Williams, M. B., Krupinski, E. A., Strauss, K. J., Breeden III, W. K., Rzeszotarski, M. S., Applegate, K., Wyatt, M., Bjork, S. \& Seibert, J. A. (2007). Digital radiography image quality: image acquisition. Journal of the American College of Radiology, 4(6), 371-388. 